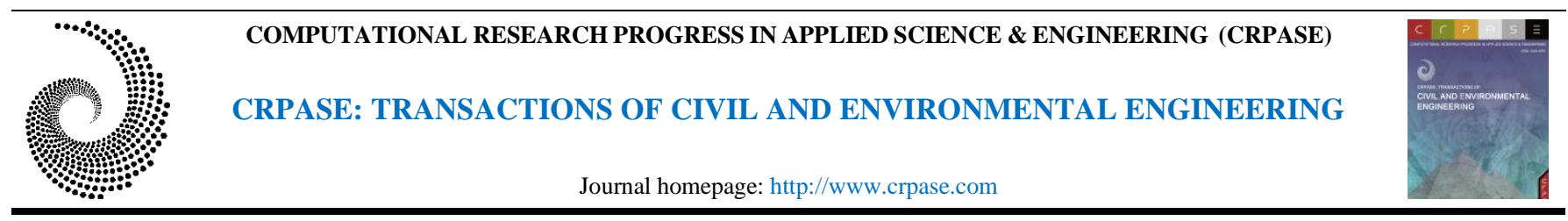

CRPASE: Transactions of Civil and Environmental Engineering 8 Article ID: 2228, 1-7, Special Issue: NCTT 2021

ISSN 2423-4591

Research Article

\title{
Contributing Factors in Pedestrian's Waiting Time at Signalized Intersections Using Survival Analysis
}

\author{
Hanieh Hashemi, Iraj Bargegol*, Gholam Hossein Hamedi \\ Department of Civil Engineering, Faculty of Engineering, University of Guilan, Rasht, Iran
}

\begin{tabular}{ll}
\hline Keywords & Abstract \\
\cline { 1 - 3 } $\begin{array}{l}\text { Pedestrian's Behavior, } \\
\text { Waiting Time, }\end{array}$ & $\begin{array}{l}\text { Recognizing elements that determine pedestrian waiting time is important when designing } \\
\text { intersection implications in order to reduce violations and their repercussions. The goal of } \\
\text { Survival Analysis, } \\
\text { Signalized Intersections. }\end{array}$ \\
$\begin{array}{l}11 \text { th pedudy is to investigate these influencing elements. To achieve this purpose, data from } \\
\text { and land uses. The T-test is performed to see if there are any significant differences between } \\
\text { sets of variables. The value of factors and the decision-making process of pedestrians are } \\
\text { determined using Survival Analysis. Pedestrians' waiting time is affected by the type of } \\
\text { median, arrival time, land use, and gender (according to survival analysis model). An } \\
\text { effective median design, based on field observations and findings, could have a considerable } \\
\text { impact on pedestrians' willingness to wait for a safe crossing during the red phase. The } \\
\text { findings of this study will aid in improving the safety of intersections by providing a better } \\
\text { understanding of the most vulnerable road users' behavior. }\end{array}$ \\
\hline
\end{tabular}

\section{Introduction}

According to the World Health Organization, there has been no decrease in the number of road traffic deaths in any lowincome country since 2013, with rates of 20.7 deaths per 100,000 population in South-East Asia, with more than half of them occurring among vulnerable road users such as pedestrians, cyclists, and motorcyclists. Many recent studies have examined the effect of pedestrian waiting time on the number and types of pedestrian violations and unsafe crossings, taking into account important factors like group size, pedestrian flow, pedestrian signal, and pedestrian maximum waiting time [1-3]. The results highlighted the impact of intersection clearing time on violations and percentage of unsafe crossings that occur. Gender and age are often regarded key factors; crossing time [2] and the waiting time [4] of the pedestrians varied with age and gender. Females waited almost twice the amount of time as males at the crossings [5], and older pedestrians have longer waiting time [6]. Another factor is arriving at a position further away from the approaching vehicle on the near side of the road or on the opposite side of the road. The position appeared to have a huge impact on pedestrian decision to cross or wait [7].

It also has been noted that as the group size increases, the proportion of violations decreases [5, 8, 9]. The number of pedestrians waiting at the corner showed a similar pattern. The more pedestrians waiting at an intersection when they arrive, the more pedestrians will join them after arrival; also, the larger the group approaching an intersection, the less likely people will break traffic laws [10].

H. Guo [6] found that the crossing location or land use has an effect on the waiting time. The study looked at three

* Corresponding Author: Iraj Bargegol
E-mail address: Bargegol@ guilan.ac.ir

Received: 17 November 2021; Revised: 11 December 2021; Accepted: 13 January 2022

https://doi.org/10.52547/crpase.8.2228

Academic Editor: Mahdi Feizbahr

Please cite this article as: H. Hashemi, I. Bargegol, G. H. Hamedi, Contributing Factors in Pedestrian's Waiting Time at Signalized Intersections Using Survival Analysis, Computational Research Progress in Applied Science \& Engineering, CRPASE: Transactions of Civil and Environmental Engineering 8 (2022) 1-7, Article ID: 2228. 
different types of land use: residential, commercial, and educational/office. Pedestrians in the educational/office area obey traffic rules at a fair level, while in the residential and industrial areas, conditions are far from satisfactory, and many pedestrians break traffic rules after a short waiting period. It's worth noting that pedestrians who are commuting for work or school are more likely to accept shorter wait times. Recent research has looked at not only how to investigate waiting times and violations, but also how to identify the different types of violations. J. Supernak [11] classified every pedestrian's intersection crossing behavior as one of the four following situations: non-violation; legal entry and legal exit, illegal entry but legal exit, legal entry but illegal exit, illegal entry, and illegal exit.

Many studies have emphasized the importance of countdown signals [12-15]. A countdown display decreases the total number of violations by a statistically significant amount [16]. Pedestrians were more likely to violate conventionaltype signals than countdown-type signals [17]. Pedestrians in China were more likely to violate conventional-type signals than countdown-type signals [17]. Furthermore, as opposed to no countdown display, the countdown signals are conducive to lowering the violation rate [18]. Personality, education, and profession were among the human characteristics examined. Other significant external factors included traffic rules and regulations, signal type, trip purpose, weather conditions, and lighting conditions [17, 19]. It seems that pedestrians are more willing to wait for the traffic signal in darkness $[9,20]$. They are more conscious on rainy days [21]. Whereas some are more likely to violate traffic rules in bad weather $[9,22]$. The study of [23] aimed to determine the relationship between pedestrian crash severity, weather, and potential risk factors, and found that the interaction effects of weather on the relationship between pedestrian crash severity, driver, and pedestrian behaviors were important. Using an umbrella in rainy weather has also been discovered to be an influential factor on pedestrians' behavior [24].

When a pedestrian is commuting through an intersection, the time of day can influence his/her decision. Pedestrians who travel in peak hours are 1.190 times more likely to end the waiting duration and cross illegally [6]. It is also important where the pedestrian is standing; curbside or median. The waiting time before crossing is affected by the crossing point [2]. M. Hamed [8] found a negative correlation between waiting time of the pedestrians before crossing the first and the second stage of the road divided by an island. T. Rosenbloom conducted a partial replication of earlier research, testing pedestrians' waiting time before crossing a third successive crosswalk and showed that Pedestrians' waiting time reduces each time [5].

\section{Methodology}

This section includes a schematic figure of the case study, followed by a brief clarification of the research process and, finally, an introduction to the applied statistical methods. Figure 1 depicts the entire process of the current study to reach the final destinations. Figure 2 (a) depicts the detailed characteristics that were taken into account during data collection. The position of pedestrians (near or far from the vehicle queue) is another category depicted in Figure 2 (b).

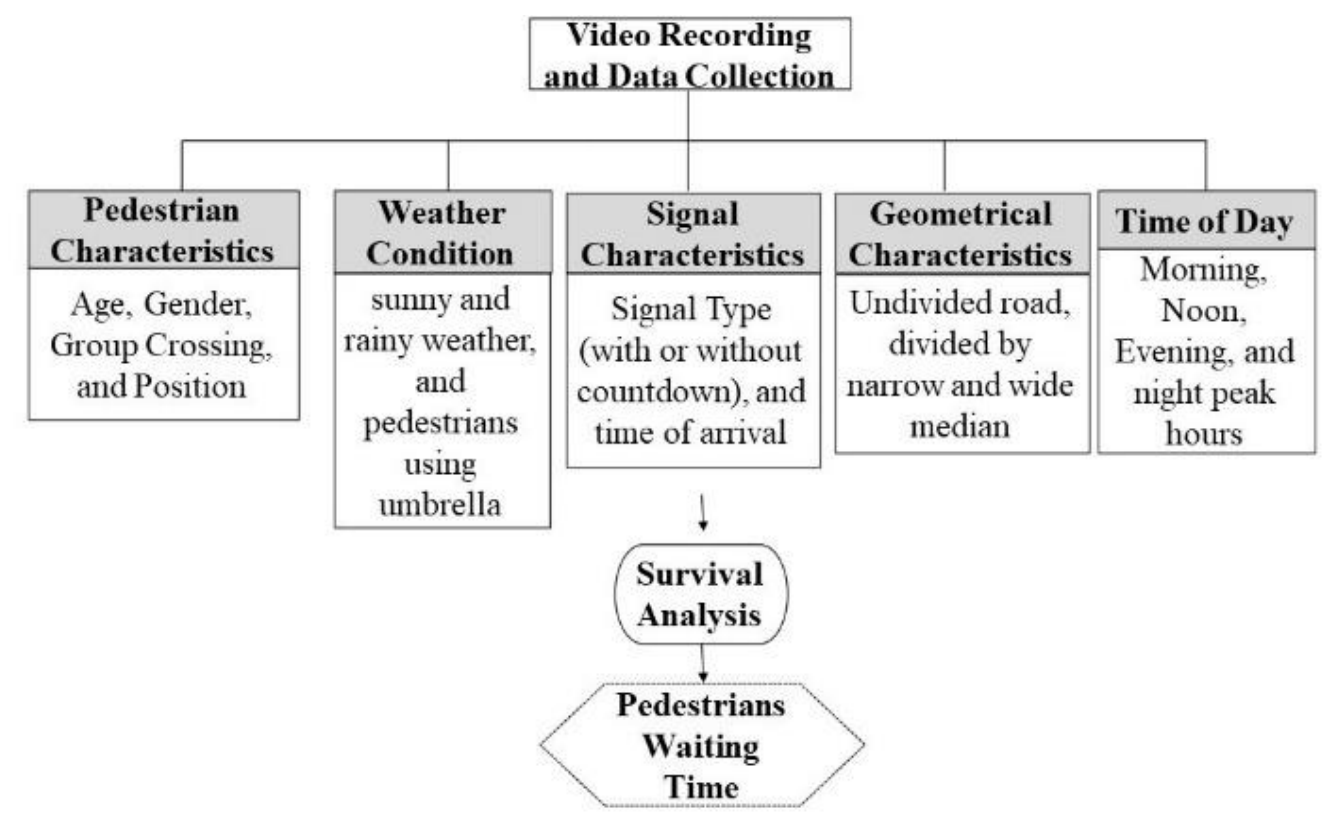

Figure 1. The flowchart of the research process 

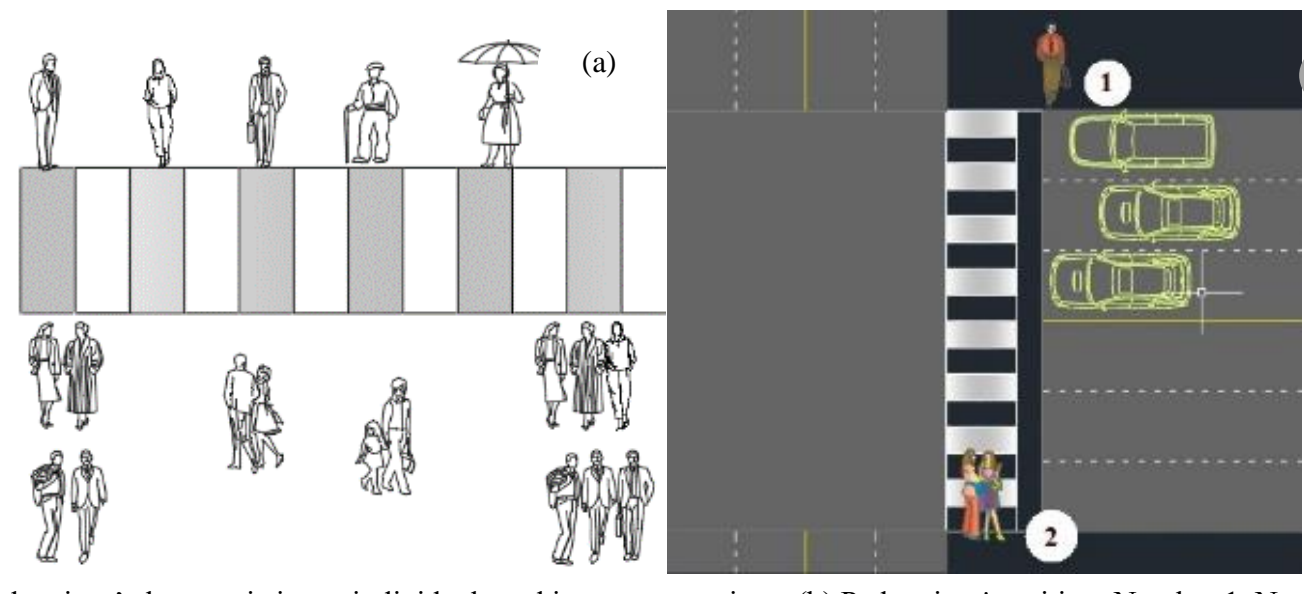

Figure 2. (a) Pedestrians' characteristics as individuals and in group crossings, (b) Pedestrians' position; Number 1. Near to the vehicle queue, and number 2 . Far from the vehicle queu

The quantitative, primary, and descriptive methods were used to obtain data from 1116 pedestrians. Group crossings are divided into three categories based on their size and gender: two people of the same gender, two people of mixed gender, and more than two people. There are four conditions depending on whether a pedestrian arrives and decides to cross, as well as when he/she completes the crossing. In this case, the definition of [11] has been modified to fit the case study in this paper:

- Fully legal crossing: a pedestrian approaches the red phase, waits until the red phase ends, and then crosses (referred to as "Legal").

- Illegal entry legal exit crossing- pedestrian arrives at the red phase, hesitates or not, begins his/her movement at the red phase, but the phase shifts somewhere in the middle, and the crossing ends at the green phase (called "rather Illegal").

- Fully illegal crossing: a pedestrian arrives and crosses during the red phase (referred to as "illegal").

- Legal entry and illegal crossing- when a pedestrian notices the signal timer is in the yellow phase but continues to cross despite the fact that there isn't enough time to make a full legal crossing (dangerous Legal)
If a pedestrian waits in the median until the next green phase on a divided lane, the crossing is considered legal.

\subsection{Case Study}

Regarding field observations, three intersections with the following characteristics were chosen:

- Three different signal type: conventional, with and without countdown timer in red and green phases.

- Divided roads with medians (narrow and wide) as well as undivided roads (figure 3)

- Traffics directed by the police (not pedestrians)

- Have a wide field of view; the camera was located on the first floor of a building with a peripheral vision on the intersections

- Two lanes in either direction, with no parked cars.

- the red phase of a traffic light lasts between 45 and 80 seconds on average, and up to 120 seconds under unusual circumstances

All the intersections are located in Rasht, Iran.

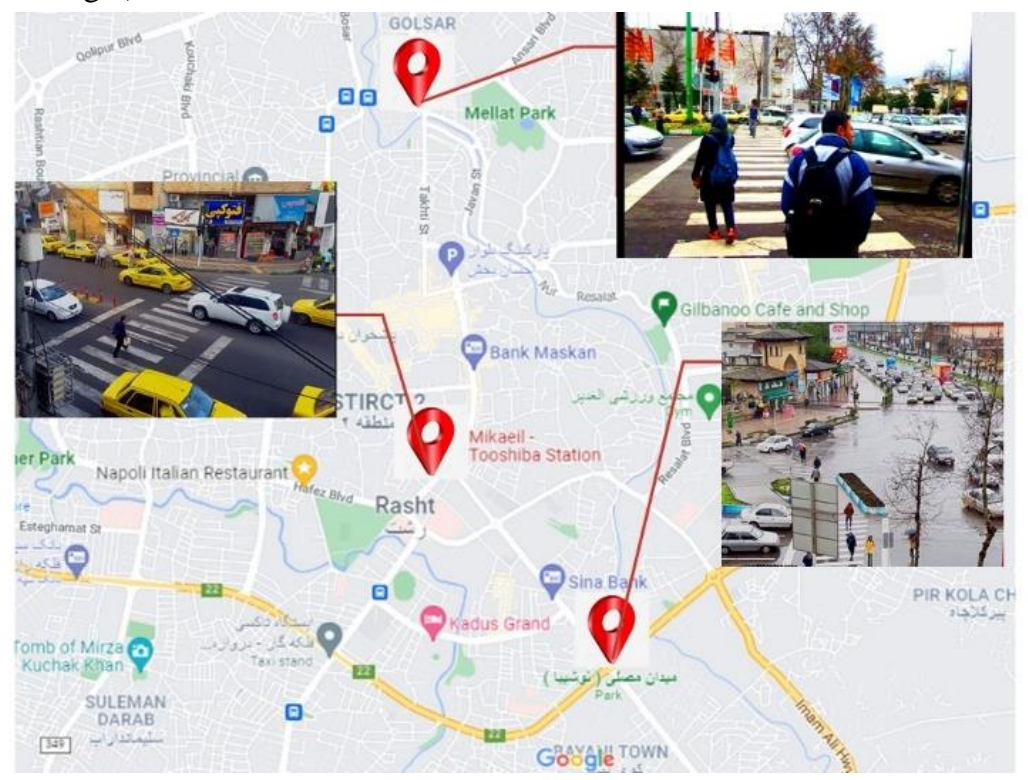

Figure 3. Geometrical condition of selected intersections with different type of medians in each leg 


\subsection{Statistics}

The information gathered was inserted into SPSS as variables, including; gender, age, land-use, signal type, group size, weather, time of day, arrival time, position, and holding an umbrella. Table 1 shows the coding process. Each group's data was normally distributed.

Table 1. Variables coding in regression models

\begin{tabular}{|c|c|c|}
\hline Category & Variable & Definition and coding \\
\hline \multirow{3}{*}{$\begin{array}{l}\text { pedestrian } \\
\text { characteristic }\end{array}$} & Gender $(\mathrm{g})$ & Male: $0 ;$ Female: 1 \\
\hline & Age (a) & Teenager: a1; Young: $a_{2}$; Old: $a_{3}$ \\
\hline & Position (p) & Whether pedestrians are near the vehicles queue or far from them; Near: 0; Far: 1 \\
\hline \multirow{2}{*}{$\begin{array}{l}\text { signal } \\
\text { characteristic }\end{array}$} & Arrival time (r) & The moment which pedestrian entrance is not legal: Clearance phase: $r_{1} ;$ first $\frac{1}{3}: r_{2} ;$ middle $\frac{1}{3}: r_{3} ;$ last $\frac{1}{3}: r_{4}$ \\
\hline & Countdown (s) & With and without countdown: 0 and 1 \\
\hline land-use & Intersection (i) & Commercial: i1; Medical: i2; Educational: i3 \\
\hline time of day & Time $(\mathrm{t})$ & Morning: $\mathrm{t}_{1} ;$ Noon: $\mathrm{t}_{2} ;$ Evening: $\mathrm{t}_{3} ;$ Night: $\mathrm{t}_{4}$ \\
\hline $\begin{array}{l}\text { geometrical } \\
\text { characteristic }\end{array}$ & Median (m) & Undivided: 0 ; median (narrow or wide): 1 \\
\hline weather & Weather $(\mathrm{w})$ & Sunny: 0; Rainy:1 \\
\hline
\end{tabular}

Survival analysis has been used to model pedestrian waiting time. The Cox regression model extends survival analysis methods to assess simultaneously the effect of several risk factors on survival time, which works for both quantitative predictor variables and for categorical variables [25]. Mathematically, the Cox model is written as $h(t)=h_{0}(t)$ $\times \exp \left\{b_{1} x_{1}+b_{2} x_{2}+\ldots+b_{p} x_{p}\right\}$, where the hazard function $h(t)$ is dependent on (or determined by) a set of $\mathrm{p}$ covariates $\left(\mathrm{x}_{1}, \mathrm{x}_{2}, \ldots, \mathrm{x}_{\mathrm{p}}\right)$, whose impact is measured by the size of the respective coefficients $\left(b_{1}, b_{2}, \ldots, b_{p}\right)$. the term $h_{0}$ is called the baseline hazard, and is the value of the hazard if all the $\mathrm{x}_{\mathrm{i}}$ are equal to zero (the quantity exp (0) equals 1 ). The ' $t$ ' in $h(t)$ reminds that the hazard may (and probably will) vary over time [26].

\section{Results and Discussions}

The position appeared to have a huge impact on pedestrian decision to cross or wait, according to [7]. Men who crossed further away from approaching vehicles (at the intersection's entrance) had a lower compliance rate in the current study. In rainy weather, the same trend has recurred, except that men's violation rate has risen more than in sunny weather. When looking at intersection land-uses separately, men who are crossing near approaching vehicles have a higher violation rate in educational land-use when it is raining. The interesting observation is that in both weather conditions, women who arrive near approaching vehicles have the lowest violation rate at all intersections. Women seem to be more vigilant when they are close approaching vehicles or a vehicle queue.

Middle-aged women (older ladies) had the most 'Legal' crossings and the fewest 'Illegal' crossings, according to the findings. Young men, on the other hand, tend to have the highest number of 'illegal' crossings between gender, age, and group categories. According to intersection land-uses, the commercial land-use with conventional signal has the most "illegal" crossings, followed by the medical land-use. On the other side, the intersection with commercial land-use has the fewest 'illegal' crossings (including dangerous ones), with a conventional signal.

There were pedestrians who arrived during the red phase and have waited until the green phase to occur. They kept 26.28 percent of all pedestrians who arrived during the red phase. Each individual's waiting time has been measured (figure 4). The average wait time for these people is 28.11 seconds.

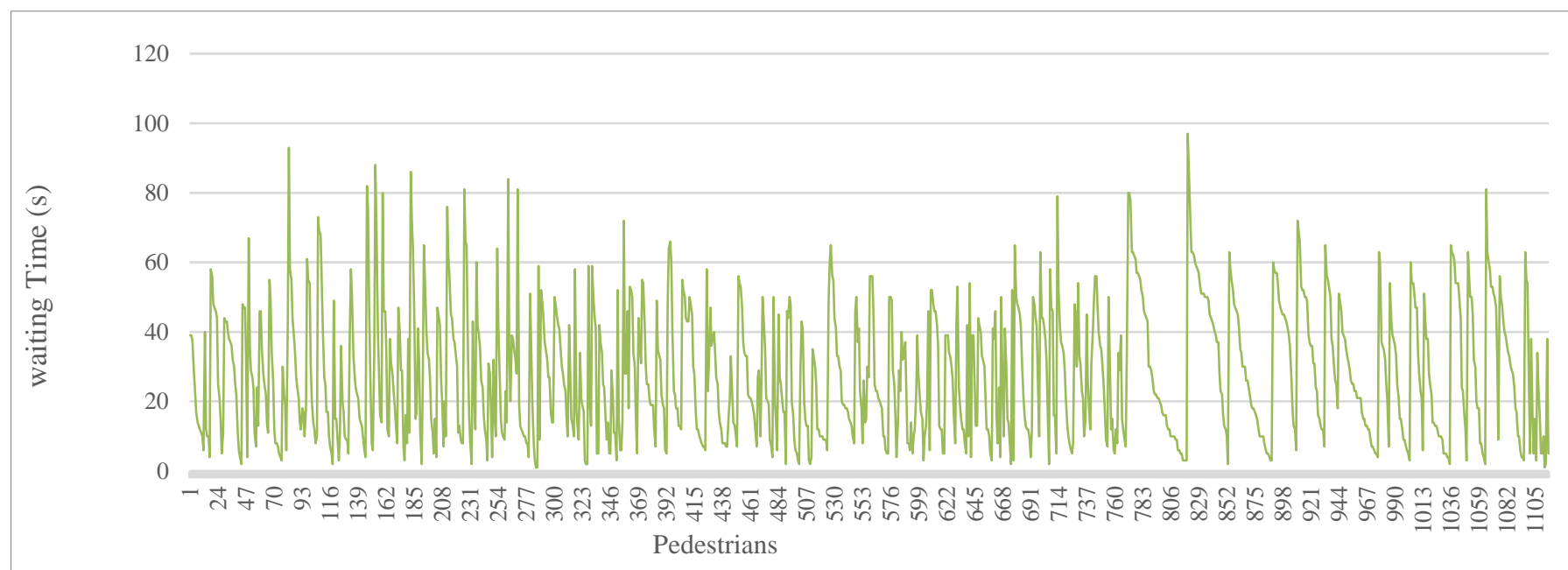

Figure 4. Each of pedestrians' waiting time 
The average waiting times at each of the three intersections show that pedestrians have waited longer in the educational land-use (figure 5) with a conventional signal. In the median of this intersection, there has been a higher amount of waiting time (>60s).

In sunny weather, the average pedestrian waiting time is 28.12 seconds, and in rainy weather, it is 28.04 seconds. According to the t-test, there is no statistically significant difference in waiting times between the two weather conditions (P-value $=0.95)$, but there is a statistically significant difference in violation rates $(\mathrm{P}$-value $<0.001)$, and pedestrian violations decrease in rainy conditions. The intersection with educational land-use (figure 5), which has a wide median, has the highest average of waiting time in the median. That may be due to the fact that the majority of users are students. The maximum average waiting time on the side of the intersection during the night's peak hour is 36 seconds due to peak hours on weekdays (6-8 p.m.). The maximum average waiting time for median is 40 seconds, which corresponds to the noon peak hour (12:30-13:30). By the way, almost a quarter of all pedestrians waited less than 12 seconds, half of all pedestrians waited less than 25 seconds, and about a quarter of all pedestrians waited more than 43 seconds for the signal to turn green. The most common waiting time for pedestrians is ten seconds.

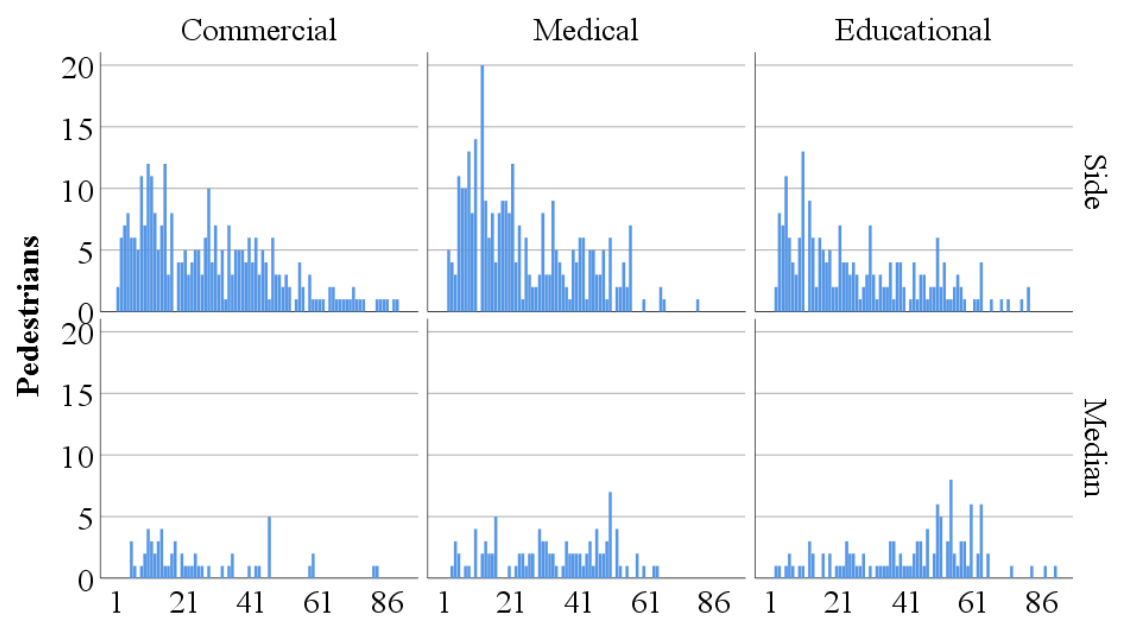

Figure 5. Pedestrians' waiting time based on the land-use, and where they are standing

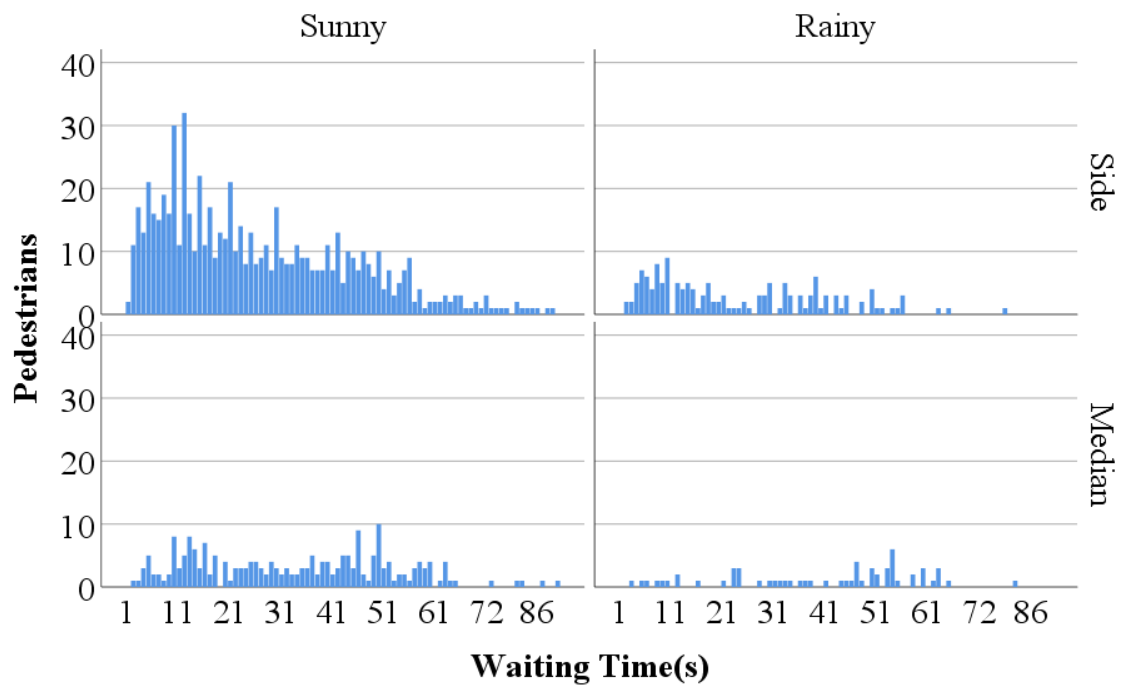

Figure 6. Pedestrians' waiting time based on weather condition, and where they are standing

\section{1. $t$-test Results}

The relevant results of comparisons between each group were discussed in the previous parts. Table 2 shows a more quantitative comparison of dual factors using the t-test, with $\mathrm{P}$-Values $<0.05$ confirming significance. The description column is created using the mean difference amounts between variables. 
Table 2. Independent Samples T-Test Results

$\begin{array}{ccc}\text { Category } & \text { Mean } & \text { Description } \\ \text { Difference } & \end{array}$

\begin{tabular}{|c|c|c|c|c|c|}
\hline \multirow{2}{*}{ Gender } & \multirow{2}{*}{\multicolumn{2}{|c|}{$\begin{array}{c}\text { Men } \\
\text { Women }\end{array}$}} & \multirow{2}{*}{$<0.001$} & \multirow{2}{*}{41.83} & \multirow[t]{2}{*}{ Men's violation rate is more than women } \\
\hline & & & & & \\
\hline \multicolumn{2}{|c|}{ Violation Behavior in Crossing } & $\begin{array}{c}\text { Individuals } \\
\text { Groups }\end{array}$ & $<0.001$ & -56.37 & $\begin{array}{l}\text { Individuals have a higher violation rate than group } \\
\text { crossings }\end{array}$ \\
\hline \multirow{2}{*}{\multicolumn{2}{|c|}{ Weather Condition }} & Sunny & \multirow{2}{*}{0.001} & \multirow{2}{*}{121.65} & The rate of violations is higher in sunny weather \\
\hline & & Rainy & & & \\
\hline \multirow{4}{*}{ Group Crossings } & twosome & $\begin{array}{l}\text { Similar } \\
\text { Mixed }\end{array}$ & 0.091 & 8.19 & $\begin{array}{l}\text { There is a slightly difference between the violation } \\
\text { rate }\end{array}$ \\
\hline & $\begin{array}{l}\text { more than } \\
\text { twosome }\end{array}$ & $\begin{array}{l}\text { Similar Group } \\
\text { Mixed Group }\end{array}$ & 0.022 & 2.33 & Similar have a higher violation rate \\
\hline & \multicolumn{2}{|c|}{ two } & & & \\
\hline & \multicolumn{2}{|c|}{ more than two } & $<0.001$ & -6.76 & $\begin{array}{l}\text { Groups including two people have a higher rate of } \\
\text { violation }\end{array}$ \\
\hline \multirow{3}{*}{ Carrying Umbrella } & & $\begin{array}{l}\text { Violation } \\
\text { Legality }\end{array}$ & 0.040 & -11.37 & $\begin{array}{l}\text { The number of legal crossings increases when } \\
\text { pedestrians hold an umbrella }\end{array}$ \\
\hline & & Men & \multirow{2}{*}{0.102} & \multirow{2}{*}{-8.89} & There is no significant difference \\
\hline & & Women & & & \\
\hline \multirow{2}{*}{ Position } & Close to the & hicles Queue & \multirow{2}{*}{$<0.001$} & \multirow{2}{*}{20.95} & The number of legal crossings increases when \\
\hline & Far from the & hicles Queue & & & pedestrians cross near the vehicles queue \\
\hline
\end{tabular}

\subsection{Survival Analysis}

According to survival curves for different groups, hazard functions are proportional and the relationship between the $\log$ hazard and each covariate is linear [27]. The coefficients of cox regression in the model of waiting time are presented in Table 3;

The model is statistically significant including four variables of the predictors. A value of $b_{i}$ greater than zero, or equivalently a hazard ratio greater than one, indicates that as the value of the ith covariate increases, the event hazard increases and thus the length of survival decreases. In fact, the hazard ratios of "gender", "arrival time", intersection", and "median", which are under 1 indicate covariates that are negatively associated with the violation probability, and thus positively associated with the length of survival or waiting time. For example, according to variables coding, for median " 0 " is undivided and " 1 " is divided with median, so the negative value will notify that the individuals that were in the decision-making process at the median were predicted to have less hazard of violation that those on the undivided road. Also, the predicted survival time will be greater in that group (code 1). Also, the variable gender is encoded as 0: male, 1: female. The exponentiated coefficients $(\exp (\mathrm{B})=$ $\exp (-0.176)=0.839)$, also known as hazard ratios, give the effect of covariates. For example, being female (gender $=1$ ) reduces the hazard by a factor of 0.83 so they have lower risk of violation (higher waiting time) than males. Weather conditions and peak hours during the day do not seem to be statistically significant in this equation.
In terms of the effects of signal type on the rate of violations and waiting time, conventional signal has a positive effect on pedestrian behavior, and higher amounts of waiting time have been recorded, similar to Guo et al. findings. Also, the recorded waiting time for countdown signal was the shortest. This can be attributed to two pieces of evidence that have been observed. First, since the intersection's land-use is commercial and the majority of trips are for shopping, pedestrians may have a lot of luggage; second, since the vehicle density is high and the speed limit is low at this intersection, pedestrians may take advantage of the opportunity to cross when they become tired or bored.

The average wait time for a legal crossing was 1.2 times longer for women than for men. It is less than the same amount in the Rosenbloom's study, which was 2 times. The 85th quantile of peak-hour waiting time at countdown equipped traffic signals is 40 seconds, which is less than Xiong's 54.4 seconds. For the conventional traffic signal, the same number is different, with 50 seconds in the current study, which is higher than Xiong's with 42.5 seconds. The percentage of pedestrian violations was similarly affected by the type of traffic signal. The rate of pedestrian violations, both dangerous and non-dangerous, was lower at the intersection with conventional signal. This is the opposite of Brosseau's observations. 
Table 3. The coefficients of cox regression in the model of waiting time

\begin{tabular}{llllll}
\hline & $\mathrm{B}$ & $\mathrm{SE}$ & Wald & Sig. & Exp(B) \\
\hline Gender & -0.176 & 0.048 & 13.653 & $<0.001$ & 0.839 \\
Arrival time & -1.256 & 0.04 & 994.795 & $<0.001$ & 0.285 \\
Intersection & -0.772 & 0.072 & 116.315 & $<0.001$ & 0.462 \\
Weather & 0.161 & 0.112 & 2.069 & 0.15 & 1.175 \\
Time & 0.025 & 0.038 & 0.441 & 0.507 & 1.026 \\
Median & -0.709 & 0.076 & 87.826 & 0.001 & 0.492 \\
\hline
\end{tabular}

\section{Conclusions}

The type of median, arrival time, intersection land-use, and gender all play a role in pedestrian waiting time (according to the survival analysis). Pedestrians who are male $(56 \%)$ and young $(49.1 \%)$ are more likely to violate; they also have less waiting time (females, 29.76s, and males, 24.46s) and more immediate crossings. Pedestrians crossing through the medical land-use with conventional signal have shown an interest in waiting in the median, even though the intersection is poorly designed. It's likely due to the type of intersection's users, who aren't all healthy or stable. Pedestrians often engage in more lawful actions when holding an umbrella under rainy condition. When it rains, men's rates of violation rise while women's rates fall. The use of a conventional signal has been demonstrated to reduce the rate of violations (both dangerous and non-dangerous) while also increasing the mean waiting time. The pedestrian's waiting time does not appear to be affected by the time of day.

\section{Conflict of Interest Statement}

The authors declare no conflict of interest.

\section{References}

[1] D. Mukherjee, S. Mitra, A comprehensive study on factors influencing pedestrian signal violation behaviour: experience from Kolkata City, India. Safety science 124 (2020) 104610.

[2] K. Shaaban, D. Muley, A. Mohammed, Analysis of illegal pedestrian crossing behavior on a major divided arterial road. Transportation research part F: traffic psychology and behaviour 54 (2018) 124-137.

[3] M. Brosseau, S. Zangenehpour, N. Saunier, L. Miranda-Moreno, The impact of waiting time and other factors on dangerous pedestrian crossings and violations at signalized intersections: A case study in Montreal. Transportation research part F: traffic psychology and behaviour 21 (2013) 159-172.

[4] N.M. Zafri, A.I. Rony, N. Adri, Analysis of Pedestrian Crossing Speed and Waiting Time at Intersections in Dhaka. Infrastructures 4 (2019) 39.

[5] T. Rosenbloom, Crossing at a red light: Behaviour of individuals and groups. Transportation research part F: traffic psychology and behaviour 12 (2009) 389-394.

[6] H. Guo, Z. Gao, X. Yang, X. Jiang, Modeling pedestrian violation behavior at signalized crosswalks in China: A hazards-based duration approach. Traffic injury prevention 12 (2011) 96-103.

[7] X. Zhuang, C. Wu, and S. Ma, Cross or wait? Pedestrian decision making during clearance phase at signalized intersections. Accident Analysis \& Prevention 111 (2018) 115-124.

[8] M.M. Hamed, Analysis of pedestrians' behavior at pedestrian crossings. Safety science 38 (2001) 63-82.

[9] D. Yagil, Beliefs, motives and situational factors related to pedestrians' self-reported behavior at signal-controlled crossings. Transportation Research Part F: Traffic Psychology and Behaviour 3 (2000) 1-13.
[10] X. Yang, An accelerated failure time model for investigating pedestrian crossing behavior and waiting times at signalized intersections. Accident Analysis \& Prevention 82 (2015) 154162.

[11] J. Supernak, V. Verma, I. Supernak, Pedestrian countdown signals: what impact on safe crossing? Open Journal of Civil Engineering 3 (2013) 39.

[12] O. Keegan, M. O'Mahony, Modifying pedestrian behaviour. Transportation Research Part A: Policy and Practice 37 (2003) 889-901.

[13] L. Rothman, MS. Cloutier, AK. Macpherson, SA. Richmond, AW. Howard, Spatial distribution of pedestrian-motor vehicle collisions before and after pedestrian countdown signal installation in Toronto, Canada. Injury prevention 25 (2019) 110 115 .

[14] J.N. Schmitz, The effects of pedestrian countdown timers on safety and efficiency of operations at signalized intersections. 2011.

[15] L. Fu, N. Zou, The influence of pedestrian countdown signals on children's crossing behavior at school intersections. Accident Analysis \& Prevention 94 (2016) 73-79.

[16] K. Lipovac, M. Vujanic, B. Maric, M. Nesic, The influence of a pedestrian countdown display on pedestrian behavior at signalized pedestrian crossings. Transportation research part F: traffic psychology and behaviour 20 (2013) 121-134.

[17] H. Xiong, L. Ma, M. Ning, X. Zhao, L. Weng, The tolerable waiting time: A generalized Pareto distribution model with empirical investigation. Computers \& Industrial Engineering 137 (2019) 106019.

[18] Q. Gong, L. Xiao, and M. Xu. Pedestrian violations crossing behavior at signal intersections: A case study in Anning District of Lanzhou. in IOP Conference Series: Materials Science and Engineering. 2019.

[19] H. Tapiro, T. Oron-Gilad, Y. Parmet, The effect of environmental distractions on child pedestrian's crossing behavior. Safety science 106 (2018) 219-229.

[20] Y.-C. Liu, Y.-C. Tung, Risk analysis of pedestrians' road-crossing decisions: Effects of age, time gap, time of day, and vehicle speed. Safety Science 63 (2014) 77-82.

[21] S. Das, C.F. Manski, M.D. Manuszak, Walk or wait? An empirical analysis of street crossing decisions. Journal of applied econometrics 20 (2005) 529-548.

[22] Y. Li, G. Fernie, Pedestrian behavior and safety on a two-stage crossing with a center refuge island and the effect of winter weather on pedestrian compliance rate. Accident Analysis \& Prevention 42 (2010) 1156-1163.

[23] X. Zhai, H. Huang, NN. Sze, Z. Song, KK. Hon, Diagnostic analysis of the effects of weather condition on pedestrian crash severity. Accident Analysis \& Prevention 122 (2019) 318-324.

[24] I. Bargegol, V.N.M. Gilani, The effect of rainy weather on walking speed of pedestrians on sidewalks. Buletin Teknol Tanaman 12 (2015) 217-22.

[25] PC. Van Dijk, KJ. Jager, AH. Zwinderman, C. Zoccali, FW. Dekker, The analysis of survival data in nephrology: basic concepts and methods of Cox regression. Kidney international 74 (2008) 705-709.

[26] MJ. Bradburn, TG. Clark, SB. Love, DG. Altman, Survival analysis part II: multivariate data analysis-an introduction to concepts and methods. British journal of cancer 89 (2003) 431436. 